\title{
Wie viele Verbote brauchen wir? Der Gesetzgeber und das ärztliche Ethos
}

\author{
Wolfram Henn
}

Online publiziert: 29. Mai 2009

(C) Springer-Verlag 2009

Ist es ethisch vertretbar, eine Schwangerschaft abzubrechen, nur weil das ungeborene Kind im Erwachsenenalter mit hoher Wahrscheinlichkeit an Brustkrebs erkranken wird? Die meisten von uns werden das verneinen, und es überrascht nicht, dass diese Meinung auch in den betroffenen Familien vorherrscht [8]. Für die Huntington-Krankheit fanden im deutschen Sprachraum in den ersten fünf Jahren nach Einführung der Gendiagnostik nur 0,6\% aller Untersuchungen pränatal statt [7], Tendenz europaweit gleichbleibend niedrig [2] - offenbar sind in der Medizin nicht alle Ebenen schief.

Kurzum: Ob man Pränataldiagnostik auf spätmanifestierende Erbleiden gesetzlich verbieten soll oder nicht, mag ein treffliches Thema für medizinethische Diskussionsrunden sein - in der Realität wird sie kaum je nachgefragt. Ein Scheinproblem also? Wohl doch mehr als nur das, denn es ist zum Schauplatz einer politischen Grundsatzdebatte geworden, durch die das letzte Konsensprojekt der Großen Koalition, das Gendiagnostikgesetz [4], fast noch auf der Zielgerade gescheitert wäre. Wie so oft, wenn ethische Fragen legislativ zu klären sind, verliefen die Fronten ungefähr, aber nicht exakt zwischen den Bundestagsfraktionen.

Auf der einen Seite fanden sich die Pragmatiker, die im Einvernehmen mit den meisten Praktikern der Schwangerenberatung und fast allen betroffenen Familien ein gesetzliches Verbot vorgeburtlicher Gentests auf erblichen Brustkrebs, Huntington-Krankheit oder Zystennieren mangels objektiven Regelungsbedarfs für verzichtbar erklärten. Zudem warnten sie, dass ein Verbot rechtlich ergebnisoffener Beratung hierzulande verunsicherte Paare mit Kinderwunsch zu dubiosen Anbietern im Ausland treiben könnte. Neben ihnen standen die nicht nur weiblichen - Verfechter maximaler Autonomie, die unter feministischem Applaus aus Prinzip jedwede gesetzliche Einschränkung des Entscheidungsradius der Schwangeren als „Bevormundung“ ablehnen (aber, nota bene, dann doch vor der pränatalen Geschlechtsselektion haltmachten).

Ihnen unversöhnlich gegenüber standen diejenigen, die sich durchaus gerne als „Lebensschützer" etikettieren lassen, und unter Verweis auf die Expansion der pränatalen Chromosomenanalysen in den achtziger und neunziger Jahren nun die spätmanifestierenden Erbleiden warnend als nächsten Abschnitt des slippery slope zur Beliebigkeit vorgeburtlicher

W. Henn $(\bowtie)$

Institut für Humangenetik, Universitätsklinikum, Bau 68, 66421 Homburg/Saar, Deutschland

E-Mail: wolfram.henn@uks.eu 
„Selektion“ benannten - in diesem Begriff seine historische Konnotation mitschwingen lassend. Unter ihnen wiederum war die kleine Gruppe der Hardliner auszumachen, denen jedwede Restriktion willkommen ist, da sie sich ohnehin ein generelles Verbot von Schwangerschaftsabbrüchen nach polnisch-irischem Muster herbeiwünschen. Ihre restriktive Haltung konnten sie im Bundestag letztlich durchsetzen, wenn auch unter weithin hörbarem Zähneknischen der Pragmatiker, die an dieser Detailfrage das Gesamtprojekt nicht scheitern lassen wollten.

Ähnlich verworren ist die Ausgangslage auf der verbliebenen legislativen Baustelle, der Novellierung des Schwangerschaftskonfliktgesetzes im Zusammenhang mit Abbrüchen aus medizinischer Indikation, insbesondere nach Pränataldiagnostik. In allen fünf vorgelegten Entwürfen und Anträgen wird zunächst der Anspruch der Schwangeren - in einigen Texten ist klugerweise von Elternpaaren die Rede - auf umfassende ärztliche und psychosoziale Beratung sowie auf ausreichende Bedenkzeit zwischen Diagnosestellung und Schwangerschaftsabbruch hochgehalten. Fachgesellschaften und Bundesärztekammer sind sich hier schon lange einig: „Da (...) die Schwangere nach den Beratungen Zeit benötigt, um ihre Entscheidung sorgfältig zu bedenken, ist die Einhaltung einer solchen Bedenkzeit in der Regel erforderlich“" ([1], S. A-3016).

Nur: Ob die für die soziale Konfliktindikation schon lange im Strafgesetzbuch festgeschriebenen drei Tage Wartezeit zwischen Beratung und Abbruch lediglich eingehalten werden sollen [5] oder aber in sanktionsbewehrter Weise eingehalten werden müssen [6], daran scheiden sich in den Gesetzentwürfen die Geister der Abgeordneten - und wohl auch ihre Einstellung zu Patientenautonomie und ärztlichem Ethos.

Hinter solch konträren Positionen in Gesetzgebungsverfahren schimmern nämlich Unterschiede in den Menschenbildern von Politikern durch, die weit über die aktuellen Debatten hinausreichen. Die einen glauben an die Einsichtsfähigkeit von Patienten unter Entscheidungsdruck und an die Kraft von Argumenten in der ärztlichen Beratung. Naiv? Die anderen zweifeln genau hieran und wollen das für sie ethisch Gebotene notfalls mit Zwangsmaßnahmen durchsetzen. Anmaßend?

Nicht einmal Charles de Montesquieu, der Urvater moderner Gesetzgebung, war sich bezüglich der Balance zwischen Vertrauen in das Ethos des Individuums und legislativem Paternalismus seiner Sache sicher. „Les lois inutiles affaiblissent les lois nécessaires “ unnütze Regeln schwächen die notwendigen ([3], liv. XXIX, chap. XVI). Andererseits: „,La vertu même a besoin de limites " - sogar die Tugend braucht Grenzen ([3], liv. XI, chap. IV).

Auch als Schwangerenberater ist man zugegebenermaßen mitunter mit sich selber nicht einig. Einerseits vertraut man auf die Kraft der sprechenden Medizin, auch ohne Androhung von Bußgeldern zum verantwortbaren Konsens von Arzt und Ratsuchenden zu finden, und glaubt über genügend Fingerspitzengefühl für den Umgang mit Ausnahmesituationen zu verfügen - so sind für ein Elternpaar, das bereits sein zweites Kind mit einem spätestens kurz nach der Geburt tödlichen Fehlbildungskomplex erwartet, nach der schon seit Wochen vorab in allen Facetten durchdiskutierten Diagnose drei weitere Tage Pflichtwartezeit nicht unbedingt hilfreich. Andererseits erleichtern klare rechtliche Vorgaben manch unerquickliche Gesprächssituation - bei einer der allfälligen telefonischen Anfragen nach einer pränatalen Vaterschaftsdiagnostik dürfte künftig der Verweis auf ein gesetzliches Verbot schneller Klarheit schaffen als bloß das Zitieren ethischer Positionspapiere aus der Ärzteschaft.

Wie auch immer: Die tatsächlichen Missstände, denen es mit Gendiagnostik- und Schwangerschaftskonfliktgesetz zu begegnen gilt, waren schon lange unumstritten, vom blühenden Gentest-Kommerz im Internet bis zu eklatanten Qualitätsmängeln bei der interdisziplinären Schwangerenberatung. Mit der im Koalitionsvertrag festgeschriebenen Selbstverpflichtung, 
für beide Problemfelder in dieser Legislaturperiode die schon lange überfälligen Regelungen zu treffen, haben die Regierenden und die hinter ihnen stehenden Parlamentarier ihre Bringschuld erst teilweise beglichen.

Es ist also weiterhin Verständigungsbereitschaft im Detail gefordert, um den Fortschritt im Ganzen nicht zu gefährden. Erschwert wird der Weg zum politischen Einvernehmen aber immer dann, wenn aus einem komplexen Zusammenhang einzelne, öffentlichkeitswirksam darstellbare Einzelaspekte herausgegriffen und zum geistig-moralischen Lackmustest erklärt werden - zumal in Wahlkampfzeiten. Die parlamentarischen Anhörungen der letzten Monate haben jedenfalls in keineswegs selbstverständlicher Harmonie zwischen Politikern aller Fraktionen, Ärzteschaft und Patientenvertretern deutlich gemacht, dass zeitgemäße Regelungen zur Pränatalmedizin unverzichtbar sind, selbst wenn sich nicht in allen Einzelfragen die jeweiligen Vorstellungen von reiner Lehre durchsetzen lassen.

Wer auf schwierige Fragen einfache Antworten parat hat, liegt ohnehin meistens falsch.

\section{Literatur}

1. Bundesärztekammer (1998) Erklärung zum Schwangerschaftsabbruch nach Pränataldiagnostik. Dt Ärztebl 95:A3013-A3016

2. Decruyenaere M, Evers-Kiebooms G, Boogaerts A, Philippe K, Demyttenaere K, et al (2007) The complexity of reproductive decision-making in asymptomatic carriers of the Huntington mutation. Eur J Hum Genet 15:453-462

3. de Montesquieu C (1748) L'esprit des loix. Barillot et fils, Genève

4. Deutscher Bundestag (2008) Entwurf eines Gesetzes über genetische Untersuchungen bei Menschen (Gendiagnostikgesetz - GenDG). BT-Drucksache 16/10532

5. Deutscher Bundestag (2009) Gesetzentwurf der Abgeordneten Ina Lenke, Sibylle Laurischk, Ulrike Flach und weiterer Abgeordneter zur Änderung des Schwangerschaftskonfliktgesetzes. BT-Drucksache $16 / 11330$

6. Deutscher Bundestag (2009) Gesetzentwurf der Abgeordneten Volker Kauder, Renate Schmidt (Nürnberg), Johannes Singhammer und weiterer Abgeordneter zur Änderung des Schwangerschaftskonfliktgesetzes. BT-Drucksache 16/11106

7. Laccone F, Engel U, Holinski-Feder E, Weigell-Weber M, Marczinek K, Nolte D, et al (1999) DNA analysis of Huntington's disease: Five years of experience in Germany, Austria, and Switzerland. Neurology 53:801-829

8. Lodder LN, Frets PG, Trijsburg RW, Meijers-Heijboer EJ, Klijn JGM, Niermeijer MF (2000) Attitudes towards termination of pregnancy in subjects who underwent presymptomatic testing for the BRCA1/ BRCA2 gene mutation in The Netherlands. J Med Genet 37:883-884 